\title{
Vibrational EELS of CaTiO3-SrTiO3 Superlattices versus Layer Thickness
}

Eric Hoglund ${ }^{1}$, Jordan Hachtel ${ }^{2}$, Thomas Beechem ${ }^{3}$, Patrick Hopkins ${ }^{1}$ and James Howe ${ }^{1}$

${ }^{1}$ University of Virginia, Charlottesville, Virginia, United States, ${ }^{2}$ Oak Ridge National Laboratory, Oak Ridge, TN, Tennessee, United States, ${ }^{3}$ Sandia National Laboratories, Albuquerque, New Mexico, United States

The vibrational response of a non-metallic material is responsible for its thermal and optical properties, and it may also cause phase transformations. For example, rotation and distortion of $\mathrm{BO}_{3}$ octahedra in $\mathrm{ABO}_{3}$ perovskite oxides are caused by softening of various phonon modes, which lead to a wide variety of phases and properties [1], [2]. The ability to tailor the phonon behavior of a material thus offers the possibility to tune material properties. For example, the stability of soft modes in $\mathrm{ABO}_{3}$ superlattices (SLs) is dependent upon both layer thickness and the monolithic constituents. Tuning the soft modes via superlattice design therefore provides a system with tunable thermal and optical properties [1], [3], [4]. Here, we show this by leveraging diffraction and electron energy-loss spectroscopy (EELS) in a scanning transmission electron microscope (STEM) to probe the phases, bonding, and vibrational modes of $\mathrm{ABO}_{3}$ superlattices with nm to sub-Å resolution and correlate them with their optical response.

Specifically, we provide a systematic analysis of surface phonon polaritons ( $\mathrm{SPhP}$ ) acquired aloof from $\mathrm{CaTiO}_{3}-\mathrm{SrTiO}_{3}$ (CTO-STO) SLs having varying superlattice repeat units of one (SL1), two (SL2), four (SL4), and 27 (SL27) unit cells. The solid lines in Figure 1a show low-loss EELS acquired perpendicular to all four SLs The EEL spectra differ markedly as a function of superlattice repeat unit. While common peaks are apparent near 22 and $71 \mathrm{meV}$ for each of the superlattices, their relative strength and the emergence of modes in the 30-60 meV range indicate changes dependent upon the layered structure itself. Quantitatively, for example, Voigt fits indicate a shifting to lower energy for a mode near $71 \mathrm{meV}$ with smaller SL period (See Figure 1b). Modes at 45 and $55 \mathrm{meV}$ shifted to higher energy when the SL, then with further reduction in SL period the modes shifted to lower energy and split. Additionally, the mode-shape in the range of 39-55 meV evolves substantially as a function of superlattice period.

To better understand the reasons for these observations, the Ti and O bonds in SL4 and SL27 were investigated using core-loss EELS, as shown in Figure 2. Both the O-K and Ti-L23 edges showed a transition from distinct CTO and STO core losses in SL27, to common edges in SL4, indicating that the STO and CTO layers adopted a similar bonding character in SL4. This change in bonding relative to SL27 would result in altered vibrational states causing the variation in the observed in the EELS shown in Fig. 1. Taken together, layering of perovskites in superlattice provides a palette to design vibrational properties and thus impact thermal and optical responses.

Acknowledgment:

Microscopy was performed as part of a user proposal at the Oak Ridge National Laboratory (ORNL), the Center for Nanophase Materials Sciences (CNMS), which is a U.S. Department of Energy, Office of Science User Facility (J.A.H.). The authors thank Prof. Ramesh Ramamoorthy for providing the thin films. 

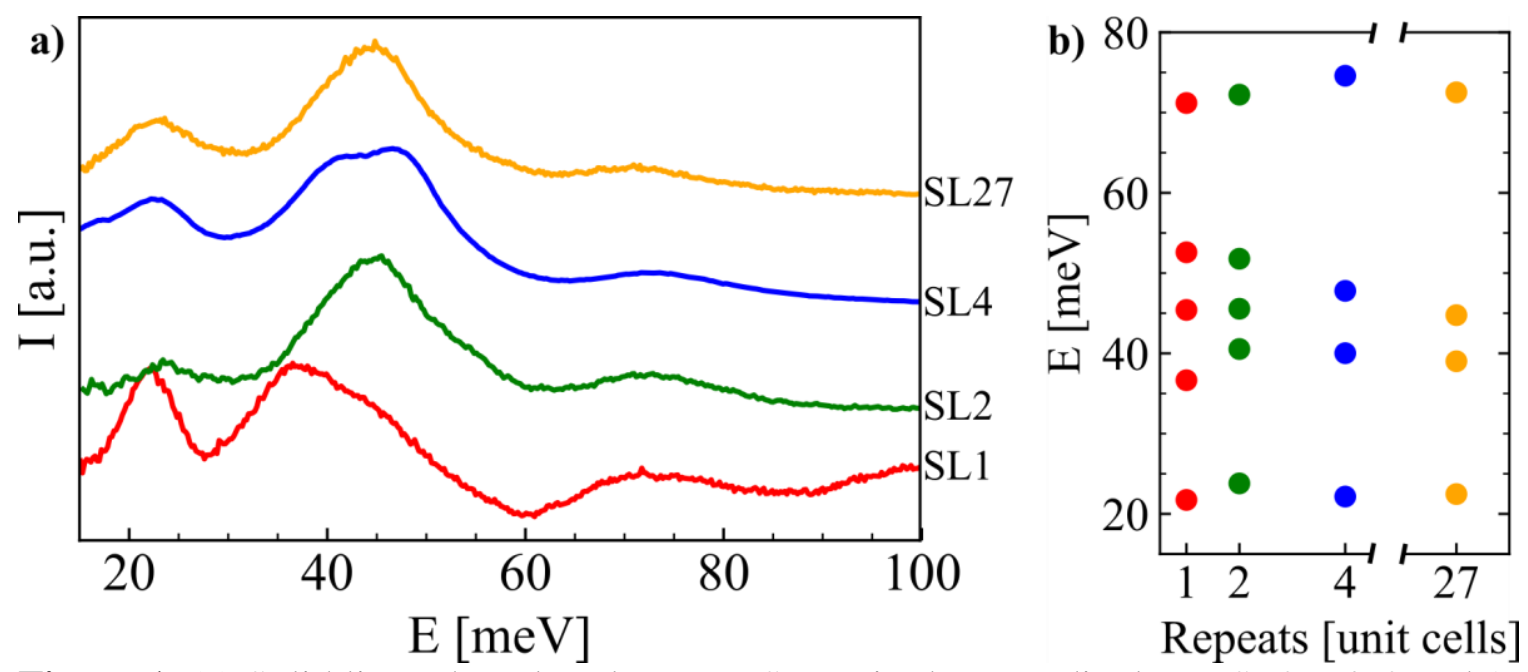

Figure 1. (a) Solid lines show low-loss EELS acquired perpendicular to SL27, 4, 2 and 1. (b) Voigt peak centers from fitted perpendicular spectra show the effect of SL layer thickness on SPhP energies.
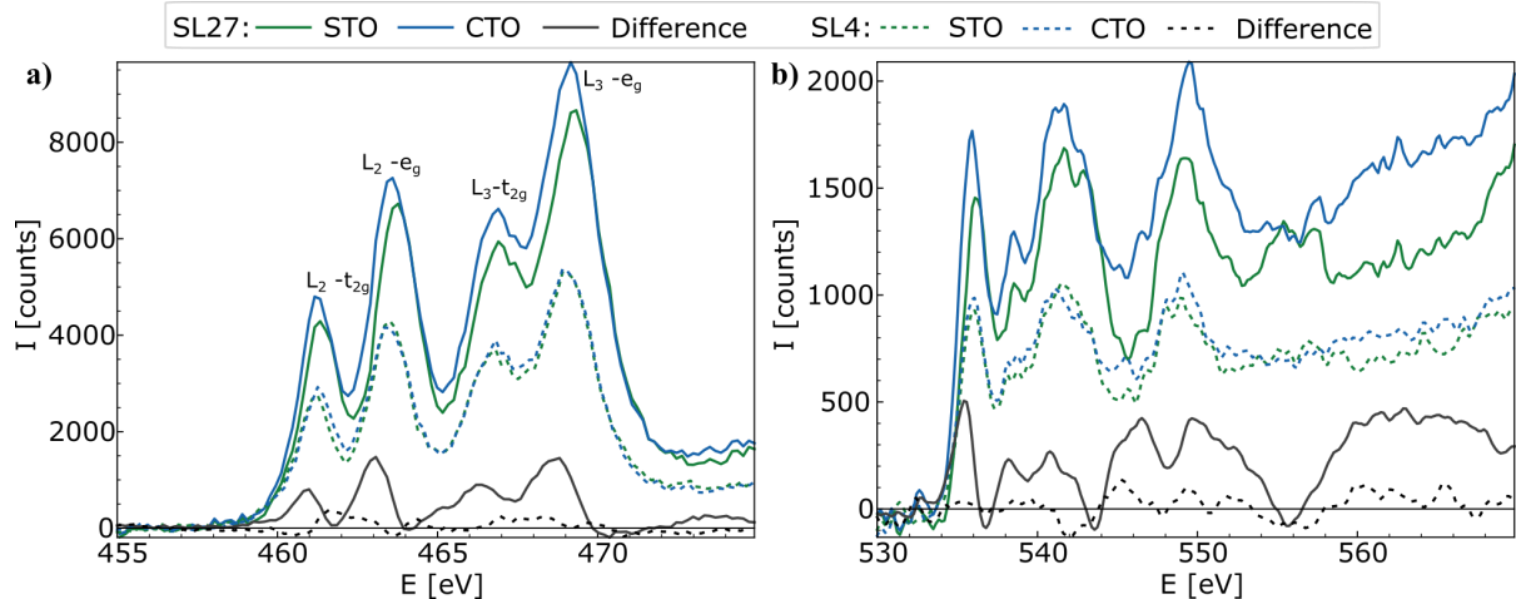

Figure 2. (a) Ti-L23 and (b) O-K edges from SL27 and 4. SL27 (solid) had distinct core edges in the CTO (blue) and STO (green), whereas SL4 (dashed) had nearly identical edges. The difference spectra (CTO minus STO) aid in identification of these similarities and dissimilarities.

\section{References}

[1] J. M. Rondinelli and C. J. Fennie, Adv. Mater., 2415 (2012), pp. 1961-1968.

[2] A. S. Bhalla, R. Guo, and R. Roy, Mater. Res. Innov. 41 (2000), pp. 3-26.

[3] H. Tabata, H. Tanaka, and T. Kawai, Appl. Phys. Lett. 6515 (1994), pp. 1970-1972.

[4] J. Ravichandran et al., Nat. Mater. 132 (2014), pp. 168-172. 